\title{
Global solution of reaction diffusion system with full matrix
}

\author{
Maroua Mebarki *, Abdelkader Moumeni \\ Departement of Mathematics,Badji Mokhtar University,Annaba-Algeria \\ *Corresponding author E-mail: marwa.mebarki@hotmail.fr
}

Copyright (c)2015 Maroua Mebarki, Abdelkader Moumeni. This is an open access article distributed under the Creative Commons Attribution License, which permits unrestricted use, distribution, and reproduction in any medium, provided the original work is properly cited.

\section{Abstract}

The purpose of this paper is to prove the global existence in time of solutions for the strongly coupled reactiondiffusion system:

$$
\begin{cases}\frac{\partial u}{\partial t}-d_{1} \Delta u-d_{2} \Delta v=f(u, v) & \text { in } R^{+} \times \Omega \\ \frac{\partial u}{\partial t}-d_{3} \Delta u-d_{4} \Delta v=g(u, v) & \text { in } R^{+} \times \Omega \\ \frac{\partial u}{\partial \eta}=\frac{\partial v}{\partial \eta}=0 & \text { in } R^{+} \times \Omega \\ u(., 0)=u_{0}(.), v(., 0)=v_{0}(.) & \text { in } \Omega\end{cases}
$$

with full matrix of diffusion coefficients. Our techniques of proof are based on Lyapunov functional methods and some $L^{p}$ estimates. we show that global solutions exist. Our investigation applied for a wide class of the nonlinear terms $f$ and $g$.

Keywords: Global Existence, Reaction Diffusion Systems, Lyapunov Functional.

\section{Introduction}

In this paper we study the following semilinear parabolic system

$$
\begin{cases}\frac{\partial u}{\partial t}-d_{1} \triangle u-d_{2} \triangle v=f(u, v) & \text { in } R^{+} \times \Omega \\ \frac{\partial v}{\partial t}-d_{3} \triangle u-d_{4} \triangle v=g(u, v) & \text { in } R^{+} \times \Omega\end{cases}
$$

Where $\Omega$ is a regular and bounded domain of $R^{n},(n \geq 1), u=u(t, x)$

$v=v(t, x), x \in \Omega, t>0$ are real valued functions, $\Delta$ denotes the Laplacian operator, and the constants of diffusion $d_{1}, d_{2}, d_{3}, d_{4}$ are assumed to be nonnegative.

System (1.1) is subjected to the following boundary conditions

$$
\frac{\partial u}{\partial \eta}=\frac{\partial v}{\partial \eta}=0 \quad \text { in } R^{+} \times \partial \Omega
$$


and the initial data

$$
u(., 0)=u_{0}(.), v(., 0)=v_{0}(.) \quad \text { in } \Omega
$$

which are assumed to be nonnegative.

The above system (1.1)-(1.3) arises in physics, chemistry and various biological processes including population dynamics. ( See [6], [23] and references therein). condition (1.2) means that there is no species of immegration .

Concerning the functions $f$ and $g$, we assume the following hypothesis:

(H1) $f(r, s)$ and $g(r, s)$ are continuously differentiable on $R^{+} \times R^{+}$, such that

$f(0, s) \geq 0, g(r, 0) \geq 0 \forall r, s \geq 0$

(H2) Assume further that there exists an integer $\forall p \geq 1$ such that

$K^{2 i-1} f(r, s)+g(r, s) \leq C(r+s+1) \quad i=1, \ldots, p$

For all $r, s \geq 0$ and a real $m \geq 1$ sach that:

$\sup (|f(r, s)|,|g(r, s)|) \leq C(r+s+1)^{m}, \forall r, s \geq 0$

The main question we want to address is the existence of global solutions for system (1.1)-(1.3). In fact the subject of the global existence of reaction diffusion systems has received a lot of attention in the last decades and several outstanding results have been proved by some of the major experts in the field. See [3, 5, 14].

This question has been investigated by many authors by considering special forms of the nonlinear terms $f$ and $g$.

In the trivial case where $d_{2}=d_{3}=d_{1}-d_{4}=0$; nonnegative solutions exist globally in time.

In diagonal case where $d_{2}=d_{3}=0$ Note that, Alikakos[1], treated the following system

$\begin{cases}u_{t}-d_{1} \triangle u=f(u, v) & \text { in } \mathbb{R}^{+} \times \Omega \\ v_{t}-d_{4} \triangle v=g(u, v) & \text { in } \mathbb{R}^{+} \times \Omega\end{cases}$

with the same boundary conditions (1.2) and initial condition (1.3), where

$f(u, v)=-g(u, v)=-u v^{\sigma}$

and gave a positive answer to the problem of the global existence of system (1.7), (1.2), (1.3) under the assumption

$1<\sigma<\sigma_{0}$

where

$\sigma_{0}=1+\frac{2}{n}$

The method used in [1] is based on some Sobolev embedding theorems.

Note that the exponent $\sigma_{0}$ given in (1.9) is exactly the critical exponents given by Fujita [7] for the parabolic problem

$\left\{\begin{array}{l}u_{t}=\triangle u+u^{\sigma} \\ u(x, 0)=u_{0}(x)\end{array}\right.$

where $u_{0}$ in (1.10) is a nonnegative. Fujita proved that if

$1<\sigma<\sigma_{0}$

then(1.10) possesses no global nonnegative solutions while if $\sigma>\sigma_{0}$, both global and nonglobal nonnegative solutions exist, depending on the nature of the initial energy. Hollis, Martin, and Pierre [10] established global existence of positive solutions for system (1.1)-(1.2) with the boundary conditions

$\lambda_{1} u+\left(1-\lambda_{1}\right) \frac{\partial u}{\partial \eta}=\beta_{1}, \lambda_{2} v+\left(1-\lambda_{2}\right) \frac{\partial v}{\partial \eta}=\beta_{2}$ on $\mathbb{R}^{+} \times \partial \Omega$ 
where

$0 \prec \lambda_{1}, \lambda_{2} \prec 1$ or $\lambda_{1}=\lambda_{2}=1$ and $\beta_{1} \succeq 0, \beta_{2} \succeq 0$

or

$\lambda_{1}=\lambda_{2}=\beta_{1}=\beta_{2}=0$

under the conditions $f(r, s)+g(r, s) \leq C(r, s)(r+s+1) ; \forall r, s \succeq 0, i=1, \ldots, p$

In [20] Masuda obtained a global existence result for a large class of the parameter $\sigma$. In fact, by using some $L^{p}$ estimates, he showed that the solution of problem (1.1)-(1.3) exists globally in time if $\sigma>1$.

The same result in [20] was obtained by Hollis et al [19] by exploiting the duality arguments on $L^{p}$ techniques, allowing to derive the uniform boundeness of the solution.

Following Masuda's approach, Haraux and Youkana [9] established a global existence result of system (1.1)-(1.3) for a large class of the function $f$ and $g$. More precisely they showed that for

$f(u, v)=-g(u, v)=-u \Phi(v)$

the problem (1.1)-(1.3) admits a global solution provided that the following condition holds:

$\lim _{(v \rightarrow+\infty)} \frac{\log (1+\Phi(v))}{v}=0$

In the general case, that is to say for

$f(u, v)=-g(u, v)$

the positivity of the function $g(u, v)$ together with the maximum principle of the heat operator give the following uniform estimate of the solution in $L^{\infty}(\Omega)$

$\|u(t)\|_{\infty} \leq\left\|u_{0}(t)\right\|_{\infty} \forall t \in\left[0, T_{\max }[\right.$

Where $T_{\max }$ is the maximal time of existence. See Pazy [24] for more details.Based on the Lyapunov functional method and for $f$ and $g$ satisfying (1.12), Kouachi [12] proved that the solution of problem (1.1)-(1.3) exists globally in time if

$\lim _{(v \rightarrow+\infty)} \frac{\log (1+f(u, v))}{v} \prec \frac{8 \alpha \beta}{n(\alpha-\beta)^{2}\left\|u_{0}\right\|_{\infty}}$

Moumeni and Salah Derradji [21] have established the existence of global solution using an approach that involves the Lyapunov's functional for the system (1.1)-(1.3) where the functions $f$ and $g$ are assumed to satisfy the condition $f(r, s)+g(r, s) \leq C(r+s+1)$.

If $d_{1} \neq d_{4}$, an important particular case is that when $f \leq 0$, which means that the first substance is absorbed by the reaction, in this case, the problem of the global existence of system (1.7) reduces to obtaining a uniform estimate for $v$, since by the maximal principle we have $u(x, t) \leq\left\|u_{0}\right\|_{\infty}$.

Here the global existence when $d_{1} \succ d_{4}$ has been treated by Kanel and Kirane [12] for a bounded domain and by Martin and Pierre [14] for whole space $R^{n}$.

Still in the case $d_{1} \neq d_{4}$, but without assuming $d_{1} \succ d_{4}$, the answer is again positive to the problem of the global existence of system (1.7) under condition (1.13) and a polynomial growth assumption on $g$ :

$g(u, v) \leq C(u+v+1)^{\gamma}$,for allu,v $\geq 0$ and some $\gamma \geq 1$,see [10] for more details.

If the diffusion coefficients are the same, that is, if $d_{1}=d_{4}$, then system (1.7) has a global solution under the condition

$f(u, v)+g(u, v) \leq 0$

,which is known as the mass dissipative structure condition. Indeed if

$d_{1}=d_{4}$, then the solution $(u, v)$ of (1.7) satisfies (by summing up the two equations in (1.7)) 
$\frac{\partial(u+v)}{\partial t}-d_{1}(u+v)=f+g \leq 0$

Then the maximal principle implies

$0 \leq u+v \leq\left\|u_{0}\right\|_{\infty}+\left\|v_{0}\right\|_{\infty}$

Therefore, the global existence follows.

In tridiagonal case where $d_{3}>0$ and $d_{2}=0$, Moumeni and Salah Derradji [22] have established the existence of global solution of the problem (1.1)-(1.3) using the Lyapunov method combined with some $L^{p}$ estimates.

For $d_{3}>0$ and $d_{2} \succ 0$ In [12] J. I. Kanel and M. Kirane proved the global existence of solutions for a strongly coupled reaction-diffusion system with homogeneous Neumann boundary conditions and

$f(u, v)=-g(u, v)=u v^{m}, m \succ 0$

$m$ is an odd integer, Later they improved their results in [13] where they obtained the global existence with

$f(u, v)=-g(u, v)=u F(v)$

On the same direction, S. Kouachi [17] has proved the global existence of solutions for two-component reactiondiffusion systems with a general full matrix of diffusion coefficients, nonhomogeneous boundary conditions and polynomial growth conditions on the nonlinear terms and he obtained in [18] the global existence of solutions for the same system with homogeneous Neumann boundary conditions and

$g(u, v)=\rho F(u, v), f(u, v)=-\sigma F(u, v) \rho \succ 0, \sigma \succ 0$

B. Rebiai and S. Benachour[25]treat the case of a general full matrix of diffusion coefficients with the homogeneous boundary conditions with nonlinearities of exponentiel growth .

finally in[4] K. Boukerrioua generalize a result obtained in [22]. Our techniques are based on invariant regions and Lyapunov functional methods.

In the present work we consider problem (1.1)-(1.3) with $d_{2}>0$ and $d_{3}>0$, where the function $f$ and $g$ are assumed to satisfy the condition (1.6), and by adopting the Lyapunov method combined with some $L^{p}$ estimates we establish a global existence result of the solution .

The content of this paper is as follows. In section 2, we introduce some notations and give a local existence result. Our main result is stated in section 3 .

\section{Local existence}

Throughout this work, we denote by $\|\cdot\|_{p}, p \in[1 ;+\infty)$ the norm in $L^{p}$ and $\|\cdot\|_{\infty}$ the norm in $C(\bar{\Omega})$ or $L^{\infty}$, respectively, defined by $\|u\|_{p}=\int_{\Omega}|u|^{p} d x^{\frac{1}{p}}$ and $\|u\|_{\infty}=e s s \sup _{x \in \Omega}|u(x)|$

The study of local existence and uniqueness of solutions $(u ; v)$ of (1.1)-(1.3) follows from the basic existence theory for parabolic semi linear equations (see, e.g., [2], [10], [24] and [27]). As a consequence, for any initial data in $L^{\infty}$ there exists a $T_{\max } \in(0 ;+\infty]$ such that (1.1)-(1.3) has a unique classical solution on $\left(0, T_{\max }[\times \Omega\right.$. Furthermore,

if $T_{\max } \prec \infty$ then $\lim _{t \rightarrow T_{\max }}\left\{\|u(t, .)\|_{\infty},\|v(t, .)\|_{\infty}\right\}=+\infty$

Therefore, if there exists a positive constant $C$ such that

$\|u(t, .)\|_{\infty}+\|v(t, .)\|_{\infty} \preceq C \forall t \in\left[0, T_{\max }\right)$ then $T_{\max }=+\infty$

Remark2.1

Under condition (H1), it follows from the invariant region method that system (1.1)-(1.3) preserves positivity. In other words, if the initial data $u_{0}$ and $v_{0}$ in (1.3) are nonnegative, then the functions $u$ and $v$ of the corresponding solution of $(1.1)-(1.3)$ are also nonnegative on $] 0, T_{\max }[\times \Omega$. See [10].

\section{Statement of the main results}

\subsection{Existence of global solutions}

In this section, we state and prove our global existence result of system (1.1)-(1.3). Our main theorem reads as follows. 


\section{Theorem3.1}

Let $p \succ \frac{m n}{2}$. Assume that condition (H2) are satisfied. Then the solution $(u(t,),. v(t,)$.$) of (1.1)-(1.3)$ with initial positive condition in $L^{\infty}(\Omega)$ exists globally in time.

We note that to prove Theorem 3.1 it is sufficient to derive a uniform estimate of $\sup \left(\|f(u, v)\|_{q},\|g(u, v)\|_{q}\right)$ for some $q>n / 2$. (See [10] for more details).

The following lemma is a useful tool in the proof of the Theorem 3.1.

\section{Lemma3.1}

Let $(u(t,),. v(t,)$.$) be the solution of (1.1)-(1.3) and let L(t)=\int_{\Omega} \sum_{i=0}^{p} C_{p}^{i} K^{i^{2}} u^{i} v^{p-i} d x$ wih $p$ a positive integer and $K$ is a serie of positive numbers such that $K \succeq \max \left(\frac{d_{1}+d_{4}}{2 \sqrt[1]{d_{1} d_{4}}}, \frac{d_{2}+d_{3}}{2 \sqrt[1]{d_{3} d_{2}}}\right)$

then the functional $L$ is uniformly bounded on the interval $\left[0, T^{*}\right] T^{*} \preceq T_{\max }$

Proof

Differentiating $L$ with respect to t yields

$$
\begin{aligned}
L^{\prime}(t)= & \int_{\Omega}\left[\sum_{i=1}^{p}\left(i C_{p}^{i} K^{i^{2}} u^{i-1} v^{p-i}\right) u_{t}+\sum_{i=0}^{p-1}\left((p-i) C_{p}^{i} K^{i^{2}} u^{i} v^{p-i-1}\right) v_{t}\right] d x \\
= & \int_{\Omega} \sum_{i=1}^{p}\left(i C_{p}^{i} K^{i^{2}} u^{i-1} v^{p-i}\right)\left(d_{1} \triangle u+d_{2} \triangle v+f(u, v)\right) d x+ \\
& \int_{\Omega} \sum_{i=0}^{p-1}\left((p-i) C_{p}^{i} K^{i^{2}} u^{i} v^{p-i-1}\right)\left(d_{3} \triangle u+d_{4} \triangle v+g(u, v)\right) d x
\end{aligned}
$$

A simple computation leads

$$
\begin{aligned}
L^{\prime}(t)= & \int_{\Omega} \sum_{i=1}^{p}\left(i C_{p}^{i} K^{i^{2}} u^{i-1} v^{p-i}\right)\left(d_{1} \triangle u+d_{2} \triangle v+f(u, v)\right) d x+ \\
& \int_{\Omega} \sum_{i=1}^{p}\left((p-i+1) C_{p}^{i-1} K^{(i-1)^{2}} u^{i-1} v^{p-i}\right)\left(d_{3} \triangle u+d_{4} \triangle v+g(u, v)\right) d x
\end{aligned}
$$

From the above equality, it follows that

$$
\begin{aligned}
L^{\prime}(t)= & \int_{\Omega} \sum_{i=1}^{p} d_{1} i C_{p}^{i} K^{i^{2}} u^{i-1} v^{p-i} \triangle u d x+\int_{\Omega} \sum_{i=1}^{p} d_{4}(p-i+1) C_{p}^{i-1} K^{(i-1)^{2}} u^{i-1} v^{p-i} \triangle v d x \\
& +\int_{\Omega} \sum_{i=1}^{p} d_{2} i C_{p}^{i} K^{i^{2}} u^{i-1} v^{p-i} \triangle v d x+\int_{\Omega} \sum_{i=1}^{p} d_{3}(p-i+1) C_{p}^{i-1} K^{(i-1)^{2}} u^{i-1} v^{p-i} \triangle u d x \\
& +\int_{\Omega} \sum_{i=1}^{p} i C_{p}^{i} K^{i^{2}} u^{i-1} v^{p-i} f(u, v) d x+\int_{\Omega} \sum_{i=1}^{p}(p-i+1) C_{p}^{i-1} K^{(i-1)^{2}} u^{i-1} v^{p-i} g(u, v) d x \\
& I+J+H
\end{aligned}
$$

By a simple use of Green's formula we have:

$I=-\int_{\Omega}\left(A|\nabla u|^{2}+B \nabla u \nabla v+C|\nabla v|^{2}\right) d x$

where:

$A=\sum_{i=2}^{p} d_{1} i(i-1) C_{p}^{i} K^{i^{2}} u^{i-2} v^{p-i}$ 
$B=\sum_{i=1}^{p-1} d_{1} i(p-i) C_{p}^{i} K^{i^{2}} u^{i-1} v^{p-i-1}+\sum_{i=2}^{p} d_{4}(i-1)(p-i+1) C_{p}^{i-1} K^{(i-1)^{2}} u^{i-2} v^{p-i}$

$C=\sum_{i=1}^{p-1} d_{4}(p-i)(p-i+1) C_{p}^{i-1} K^{(i-1)^{2}} u^{i-1} v^{p-i-1}$

Using the fact that :

$i C_{p}^{i}=(p-i+1) C_{p}^{i-1}=p C_{p-1}^{i-1} \quad \forall i=1, \ldots, p$

and also since

$i(i-1) C_{p}^{i+1}=i(p-i) C_{p}^{i}=(p-i)(p-i+1) C_{p}^{i-1}=p(p-1) C_{p-2}^{i-2}$

we get

$A=\sum_{i=2}^{p} d_{1} p(p-1) C_{p-2}^{i-2} K^{i^{2}} u^{i-2} v^{p-i}$

$B=\sum_{i=1}^{p-1} d_{1} p(p-1) C_{p-2}^{i-2} K^{i^{2}} u^{i-1} v^{p-i-1}+\sum_{i=2}^{p} d_{4} p(p-1) C_{p-2}^{i-2} K^{(i-1)^{2}} u^{i-2} v^{p-i}$

$=B_{1}+B_{2}$

and

$C=\sum_{i=1}^{p-1} d_{4} p(p-1) C_{p-2}^{i-1} K^{(i-1)^{2}} u^{i-1} v^{p-i-1}$

Putting : $j=i-2$, we have :

$A=\sum_{j=0}^{p-2} d_{1} p(p-1) C_{p-2}^{j} K^{(j+2)^{2}} u^{j} v^{p-j-2}$

$B_{2}=\sum_{j=0}^{p-2} d_{4} p(p-1) C_{p-2}^{j} K^{(j+1)^{2}} u^{j} v^{p-j-2}$

and Putting : $j=i-1$, we get :

$B_{1}=\sum_{j=0}^{p-2} d_{1} p(p-1) C_{p-2}^{j} K^{(j+1)^{2}} u^{j} v^{p-j-2}$

$C=\sum_{j=0}^{p-2} d_{4} p(p-1) C_{p-2}^{j} K^{j^{2}} u^{j} v^{p-j-2}$

Then :

$I=-p(p-1) \sum_{j=0}^{p-2} C_{p-2}^{j} \int_{\Omega} u^{j} v^{p-j-2} \times \Psi(\nabla u, \nabla v) d x$ 
where

$\Psi(\nabla u, \nabla v)=d_{1} K^{(j+2)^{2}}|\nabla u|^{2}+\left(d_{1}+d_{4}\right) K^{(j+1)^{2}} \nabla u \nabla v+d_{4} K^{j^{2}}|\nabla v|^{2}$

The quadratic forms are positive since :

$\left(\left(d_{1}+d_{4}\right) K^{(j+1)^{2}}\right)^{2}-4 d_{1} d_{4} K^{j^{2}} K^{(j+2)^{2}} \preceq 0 \quad j=0, \ldots, p-2$

Using the relation $K \succeq \max \left(\frac{d_{1}+d_{4}}{2 \sqrt[1]{d_{1} d_{4}}}, \frac{d_{2}+d_{3}}{2 \sqrt[1]{d_{3} d_{2}}}\right)$

Then

$I \preceq 0$

By a simple use of Green's formula we have:

$J=-\int_{\Omega}\left(D|\nabla v|^{2}+E \nabla v \nabla u+F|\nabla u|^{2}\right) d x$

where:

$D=\sum_{i=1}^{p-1} d_{2} i(p-i) C_{p}^{i} K^{i^{2}} u^{i-1} v^{p-i-1}$

$E=\sum_{i=2}^{p} d_{2} i(i-1) C_{p}^{i} K^{i^{2}} u^{i-2} v^{p-i}+\sum_{i=1}^{p-1} d_{3}(p-i)(p-i+1) C_{p}^{i-1} K^{(i-1)^{2}} u^{i-1} v^{p-i-1}$

$F=\sum_{i=2}^{p} d_{3}(i-1)(p-i+1) C_{p}^{i-1} K^{(i-1)^{2}} u^{i-2} v^{p-i}$

Using the relation (3.2) we get

$D=\sum_{i=1}^{p-1} d_{2} p(p-1) C_{p-2}^{i-2} K^{i^{2}} u^{i-1} v^{p-i-1}$

$\begin{aligned} E= & \sum_{i=2}^{p} d_{2} p(p-1) C_{p-2}^{i-2} K^{i^{2}} u^{i-2} v^{p-i}+\sum_{i=1}^{p-1} d_{3} p(p-1) C_{p-2}^{i-1} K^{(i-1)^{2}} u^{i-1} v^{p-i-1} \\ & E_{1}+E_{2}\end{aligned}$

and

$F=\sum_{i=2}^{p} d_{3} p(p-1) C_{p-2}^{i-2} K^{(i-1)^{2}} u^{i-2} v^{p-i}$

putting : $j=i-1$, we have :

$D=\sum_{j=0}^{p-2} d_{2} p(p-1) C_{p-2}^{j} K^{(j+1)^{2}} u^{j} v^{p-j-2}$

$E_{2}=\sum_{j=0}^{p-2} d_{3} p(p-1) C_{p-2}^{j} K^{j^{2}} u^{j} v^{p-j-2}$

and putting : $j=i-2$, we get : 
$E_{1}=\sum_{j=0}^{p-2} d_{2} p(p-1) C_{p-2}^{j} K^{(j+2)^{2}} u^{j} v^{p-j-2}$

$F=\sum_{j=0}^{p-2} d_{3} p(p-1) C_{p-2}^{j} K^{(j+1)^{2}} u^{j} v^{p-j-2}$

Then :

$J=-p(p-1) \sum_{j=0}^{p-2} C_{p-2}^{j} \int_{\Omega} u^{j} v^{p-j-2} \times \Phi(\nabla v, \nabla u) d x$

where

$\Phi(\nabla v, \nabla u)=d_{2} K^{(j+1)^{2}}|\nabla v|^{2}+\left(d_{2} K^{(j+2)^{2}}+d_{3} K^{j^{2}}\right) \nabla v \nabla u+d_{3} K^{(j+1)^{2}}|\nabla u|^{2}$

The quadratic forms are positive since :

$\left(\left(d_{2} K^{(j+2)^{2}}+d_{3} K^{j^{2}}\right)\right)^{2}-4 d_{2} d_{3} K^{(j+1)^{2}} K^{(j+1)^{2}} \preceq 0 \quad j=0, \ldots, p-2$

Using the relation $K \succeq \max \left(\frac{d_{1}+d_{4}}{2 \sqrt[1]{d_{1} d_{4}}}, \frac{d_{2}+d_{3}}{2 \sqrt[1]{d_{3} d_{2}}}\right)$

Then

$J \preceq 0$

Using the relation (3.2), in the third integral, yields :

$H=\int_{\Omega}\left[p \sum_{i=1}^{p}\left(K^{i^{2}} f(u, v)+K^{(i-1)^{2}} g(u, v)\right) C_{p-1}^{i-1} u^{i-1} v^{p-i}\right] d x$

Using the relation(1.5) we deduce

$H \preceq c_{3} \int_{\Omega}\left[\sum_{i=1}^{p}(u+v+1) C_{p-1}^{i-1} u^{i-1} v^{p-i}\right] d x$

To prove that the functional $L$ is uniformly bounded on the interval $\left[0, T^{*}\right]$ first we write

$L^{\prime}(t) \preceq c_{3} \int_{\Omega}\left[\sum_{i=1}^{p} C_{p-1}^{i-1} u^{i} v^{p-i}+\sum_{i=1}^{p} C_{p-1}^{i-1} u^{i-1} v^{p-i+1}+\sum_{i=1}^{p} C_{p-1}^{i-1} u^{i-1} v^{p-i}\right] d x$

$L^{\prime}(t) \preceq c_{3} \int_{\Omega}\left[\sum_{i=1}^{p} C_{p-1}^{i-1} u^{i} v^{p-i}+\sum_{i=0}^{p-1} C_{p-1}^{i} u^{i} v^{p-i}+\sum_{i=0}^{p-1} C_{p-1}^{i} u^{i} v^{p-i-1}\right] d x$

$L^{\prime}(t) \preceq c_{3} \int_{\Omega}\left[\sum_{i=0}^{p} C_{p}^{i} u^{i} v^{p-i}+\sum_{i=0}^{p-1} C_{p-1}^{i} u^{i} v^{p-i-1}\right] d x$

Using the fact that

$\sum_{i=0}^{p-1} C_{p-1}^{i} u^{i} v^{p-i-1}=(u+v)^{p-1}$ 
Therefore, the last inequality can be written as

$L^{\prime}(t) \preceq c_{1}(p) L(t)+c_{3} \int_{\Omega}(u+v)^{p-1}$

Applying Hôlder's inequality to the second term in the right hand side of the above inequality, we obtain

$\left.L^{\prime}(t) \preceq c_{1}(p) L(t)+c_{3}(m e s \Omega)^{\frac{1}{p}}\left(\int_{\Omega}(u+v)^{p} d x\right)^{\frac{(p-1)}{p}}\right)$

Since the following inequality holds,

$(u+v)^{p}=\sum_{i=0}^{p} C_{p}^{i} u^{i} v^{p-i} \preceq \frac{\sup _{0 \preceq i \preceq p} C_{p}^{i}}{\min _{0 \preceq i \preceq p} C_{p}^{i} K^{i^{2}}} \sum_{i=0}^{p} C_{p}^{i} K^{i^{2}} u^{i} v^{p-i}$

Then, we have

$L^{\prime}(t) \preceq c_{1}(p) L(t)+c_{3}(m e s \Omega)^{\frac{1}{p}}\left(\frac{\sup _{0 \preceq i \preceq p} C_{p}^{i}}{\min _{0 \preceq i \preceq p} C_{p}^{i} K^{i^{2}}}\right)^{\frac{(p-1)}{p}}(L(t))^{\frac{(p-1)}{p}} \quad \forall t \prec T_{\max }$

Hence, $L(t)$ the functional satisfies the following differential inequality:

$L^{\prime}(t) \preceq c_{1}(p) L(t)+c_{2}(p)(L(t))^{\frac{(p-1)}{p}} \quad \forall t \prec T_{\max }$

where

$c_{2}(p)=c_{3}(m e s \Omega)^{\frac{1}{p}}\left(\frac{\sup _{0 \preceq i \preceq p} C_{p}^{i}}{\min _{0 \preceq i \preceq p} C_{p}^{i} K^{i^{2}}}\right)^{\frac{(p-1)}{p}}$

which gives us, by a simple integration

$(L(t))^{\frac{1}{p}} \preceq\left[(L(0))^{\frac{1}{p}}+\frac{c_{2}^{\prime}(p)}{c_{1}^{\prime}(p)}\right] \exp \left(c_{1}^{\prime}(p) t\right)-\frac{c_{2}^{\prime}(p)}{c_{1}^{\prime}(p)}$

where

$c_{1}^{\prime}(p)=\frac{c_{1}(p)}{p} \quad c_{2}^{\prime}(p)=\frac{c_{2}(p)}{p}$

By using the inequality

$L(t)=\int_{\Omega}\left(\sum_{i=0}^{p} C_{p}^{i} K^{i^{2}} u^{i} v^{p-i}\right) d x \succeq \int_{\Omega}\left(C_{p}^{p} K^{p^{2}} u^{p}+C_{p}^{0} K^{0^{2}} v^{p}\right) d x$

it follows that

$L(t) \succeq \min \left(C_{p}^{0} K^{0^{2}}, C_{p}^{p} K^{p^{2}}\right) \sup \left(\int_{\Omega} u^{p} d x, \int_{\Omega} v^{p} d x\right)$

Hence,

$(L(t))^{\frac{1}{p}} \succeq\left[\min \left(C_{p}^{0} K^{0^{2}}, C_{p}^{p} K^{p^{2}}\right)\right]^{\frac{1}{p}} \sup \left(\left(\int_{\Omega} u^{p} d x\right)^{\frac{1}{p}},\left(\int_{\Omega} v^{p} d x\right)^{\frac{1}{p}}\right)$

And therefore,

$\sup \left(\|u(t, .)\|_{p},\|v(t, .)\|_{p}\right) \preceq \frac{(L(t))^{\frac{1}{p}}}{\left[\min \left(C_{p}^{0} K^{0^{2}}, C_{p}^{p} K^{p^{2}}\right)\right]^{\frac{1}{p}}} \quad \forall t \prec T_{\max }$ 
With (3.11) and (3.12) we obtain :

$\sup \left(\|u(t, .)\|_{p},\|v(t, .)\|_{p}\right) \preceq c(t) \quad \forall t \prec T_{\max }$

where

$c(t)=\frac{1}{\left[\min \left(C_{p}^{0} K^{0^{2}}, C_{p}^{p} K^{p^{2}}\right)\right]^{\frac{1}{p}}}\left\{\left[(L(0))^{\frac{1}{p}}+\frac{c_{2}^{\prime}(p)}{c_{1}^{\prime}(p)}\right] \exp \left(c_{1}^{\prime}(p) t\right)-\frac{c_{2}^{\prime}(p)}{c_{1}^{\prime}(p)}\right\}$

The proof of Lemma 3.1 is complete.

Proof of theorem3.1

From (1.6)we have

$\sup (|f(u, v)|,|g(u, v)|) \preceq c_{2}(u+v+1)^{m}$

Then, it follows that

$\sup \left(\int_{\Omega}|f(u, v)|^{\frac{p}{m}} d x, \int_{\Omega}|g(u, v)|^{\frac{p}{m}} d x \preceq c_{2}^{\frac{p}{m}} \int_{\Omega}(u+v+1)^{p} d x\right.$

which implies :

$\sup \left(\|f(u, v)\|_{\frac{p}{m}}^{\frac{p}{m}},\|g(u, v)\|_{\frac{p}{m}}^{\frac{p}{m}}\right) \preceq c_{2}^{\frac{p}{m}} \int_{\Omega}(u+v+1)^{p} d x$

On the other hand, we have

$$
\begin{aligned}
& \int_{\Omega}(u+v+1)^{p} d x=\int_{\Omega}^{k} \sum_{k=0}^{p} C_{p}^{k}(u+v)^{k} d x \\
& \int_{\Omega}(u+v+1)^{p} d x=\int_{\Omega}\left[1+(u+v)^{p}\right] d x+\sum_{k=1}^{p-1} C_{p}^{k} \int_{\Omega}(u+v)^{k}
\end{aligned}
$$

An application of Hôlder's inequality leads

$$
\sum_{k=1}^{p-1} C_{p}^{k} \int_{\Omega}(u+v)^{k} \preceq \sum_{k=1}^{p-1} C_{p}^{k}\left[\int_{\Omega}\left(1^{\frac{p}{(p-k)}} d x\right)^{\frac{(p-k)}{p}}\left(\int_{\Omega}(u+v)^{p} d x\right)^{\frac{k}{p}}\right]
$$

Hence

$$
\begin{aligned}
\int_{\Omega}(u+v+1)^{p} d x \preceq & \operatorname{mes}(\Omega)+\int_{\Omega}(u+v)^{p} d x \\
& +\sum_{k=1}^{p-1} C_{p}^{k}\left[(\operatorname{mes}(\Omega))^{\frac{(p-k)}{p}}\left(\int_{\Omega}(u+v)^{p} d x\right)^{\frac{k}{p}}\right]
\end{aligned}
$$

using (3.13) we get:

$\left(\int_{\Omega}(u+v)^{p} d x\right)^{\frac{1}{p}}=\|u(t, .)+v(t, .)\|_{p} \preceq\|u(t, .)\|_{p}+\|v(t, .)\|_{p} \preceq 2 c(t)$

and the inequality (3.15) can be written as follows

$$
\begin{aligned}
& \int_{\Omega}(u+v+1)^{p} d x \preceq \operatorname{mes}(\Omega)+2^{p}(c(t))^{p}+\sum_{k=1}^{p-1} C_{p}^{k}\left[(\operatorname{mes}(\Omega))^{\frac{(p-k)}{p}}(2 c(t))^{k}\right. \\
& \preceq \sum_{k=0}^{p} C_{p}^{k}\left[(\operatorname{mes}(\Omega))^{\frac{(p-k)}{p}}(2 c(t))^{k}\right.
\end{aligned}
$$


Therefore

$\sup \left(\left(\|f(u, v)\|_{\frac{p}{m}}^{\frac{p}{m}},\|g(u, v)\|_{\frac{p}{m}}^{\frac{p}{m}}\right) \preceq c^{\frac{p}{m}} \sum_{k=0}^{p} C_{p}^{k}\left[(\operatorname{mes}(\Omega))^{\frac{(p-k)}{p}}(2 c(t))^{k}\right.\right.$

which gives that

$\sup \left(\|f(u, v)\|_{\frac{p}{m}},\|g(u, v)\|_{\frac{p}{m}}\right) \preceq c_{p, m}(t) \quad \forall t \prec T_{\max }$

where

$c_{p, m}(t)=c\left[\sum_{k=0}^{p} 2^{k} C_{p}^{k}\left[(\operatorname{mes}(\Omega))^{\frac{(p-k)}{p}}(c(t))^{k}\right]^{\frac{p}{m}}\right.$

\section{Remark3.1}

From both Lemma 3.1 and Theorem 3.1, we have obtained an uniform estimate of $\sup \left(\|f(u, v)\|_{q},\|g(u, v)\|_{q}\right)$ with $q=p / m>n / 2$. By the preliminary remarks, we conclude that the solution of the given problem exists globally in time.

\section{References}

[1] N. Alikakos, $L^{p}$ bounds of solutions of reaction-diffusion equations, Comm. Partial Differential Equations 4 (1979), 827-828.

[2] H. Amann, Dynamic theory of quasilinear parabolic equations - I. Abstract evolution equations, Nonlinear Anal. 12 (1988), 895-919.

[3] S. Bonaved, D. Schmitt, Triangular reaction-diffusion systems with integrable initial data, Nonlinear. Anal 33 (1998), 785-801.

[4] K. Boukerrioua,existence of global solutions for a system of reaction-diffusion equations having a full matrix Ser. Math. Inform. Vol. 29, No 1 (2014), 91-103.

[5] T. Diagana, Some remarks on some strongly coupled reaction-diffusion equations, J.Reine. Angew., 2003.

[6] R. Fisher, The advance of advantageous genes, Ann. Eugenics 7 (1937), 335-369.

[7] H. Fujita, On the blowing up of solutions to the Cauchy problem for $\partial u / \partial t=\Delta u+u^{(\sigma+1)}$ J. Fac. Sci. Univ. Tokyo Sect. A Math. 16 (1966), 105-113.

[8] A. Haraux, M. Kirane, Estimation C1 pour des problèmes paraboliques semi-linéaires,Ann. Fac. Sci. Toulouse Math. 5 (1983), 265-280

[9] A. Haraux, A. Youkana, On a result of K. Masuda concerning reaction-diffusion equations, Tohoku. Math. J. 40 (1988), 159-163.

[10] D. Henry, Geometric Theory of Semilinear Parabolic Equations, Lecture Notes in Math., 840, Springer Verlag, New York, 1981.

[11] S. L. Hollis, R. H. Martin, M. Pierre, Global existence and boundedness in reaction diffusion systems, SIAM. J. Math. Anal. 18(3) (1987), 744-761.

[12] J. I. Kanel and M. Kirane, Pointwise a priori bounds for a strongly coupled system of reaction-diffusion equations with a balance law, Math. Methods Appl. Sci. 21 (1998), 1227-1232.

[13] I. Kanel, M. Kirane, Global existence and large time behavior of positive solutions to a reaction diffusion system, Differ. Integral Equ. Appl. 13(1-3) (2000), 255-264. 
[14] S. Kouachi, Global existence of solutions to reaction diffusion systems via a Lyapunov functional, Electron. J. Differential Equations (68) (2001), 1-10.

[15] S. Kouachi, Global existence of solutions for reaction-diffusion systems with a full matrix of diffusion coe cients and nonhomogeneous boundary conditions,Electron. J. Qual. Theory Di er. Equ. 4 (2002), pp. 1-10.

[16] S. Kouachi, Global existence of solutions in invariant regions for reaction-diffusion systems with a balance law and a full matrix of di usion coe cients,Electron. J. Qual. Theory Di er. Equ. 2 (2003), pp. 1-10.

[17] S. Kouachi, Invariant regions and global existence of solutions for reaction-diffusion systems with full matrix of diffusion coefficients and nonhomogeneous boundary conditions, Georgian Math. J. 11 (2004), 349-359.

[18] S. Kouachi, A. Youkana, Global existence and asymptotics for a class of reaction diffusion systems, Bull. Polish Acad. Sci. Math. 49(3), 2001.

[19] R. H. Martin, M. Pierre, Nonlinear reaction-diffusion systems, in: Nonlinear Equations in the Applied Sciences, Math. Sci. Eng. Acad. Press, New York 1991.

[20] K. Masuda, On the global existence and asymptotic behavior of solutions of reaction diffusion equations, Hokkaido Math. J. 12 (1983), 360-370.

[21] A. Moumeni, L. Salah Derradji, Global existence of solution for reaction diffusion systems, IAENG, Int. J. Appl. Math. 40(2) (2010), 84-90.

[22] A. Moumeni, L. Salah Derradji, Global existence of solution for reaction diffusion Systems with non diagonal matrix, Demonstratio Mathematica, Vol. XLV No 1( 2012 ).

[23] J. D. Murray, Mathematical Biologie, 3rd ed., Interdisciplinary Applied Mathematics,Springer Verlag, 2002.

[24] A. Pazy, Semigroups of linear operators and applications to partial differential equations, Applied Mathematical Siences, Springer-Verlag, New York, 1983.

[25] M. Pierre, D. Schmitt, Blow up in reaction-diffusion systems with dissipation of mass,SIAM. J. Math. Anal. 42(1) (2000), 93-106.

[26] B. Rebiai and S. Benachour, Global classical solutions for reactiondiffusion systems with nonlinearities of exponential growth, J. Evol. Equ.10 (2010), 511-527.

[27] F. Roth, Global solutions of reaction diffusion systems, Lecture Notes in Math. 1072,Springer Verlag, Berlin, 1984. bounds of solutions of reaction-diffusion equations, Comm. Partial Differential Equations 4 (1979), 827-828. 